Pacific Journal of Mathematics

ON. THEOREM OF L. L. SALTILY 


\title{
ON A THEOREM OF L. A. SANTALO'
}

\author{
B. GR ÜNBAUM
}

1. Introduction. L. A. Santaló [2] proved, as a by-product of other investigations, the following theorem:

Let a set of parallel line segments be given in the plane. If every three of the segments can be intersected (that is, met) by a straight line, then there exists a straight line intersecting all the segments.

This result was rediscovered by M. Dresher and T. E. Harris (cf. [1]). An interesting generalisation was obtained by H. Rademacher and I. J. Schoenberg [1]. Their generalisation-extending a special case due to T.E. Harris-is obtained from Santalo's theorem on replacing in it "three" by " $m+2$ " and "straight line" by "polynomial line $y=\alpha_{0} x^{m}+\cdots+\alpha_{m}$ ".

The proof of Rademacher and Schoenberg (as well as a proof of Santalo's original theorem by J. Rey Pastor, cf. [2]) is based on Helly's theorem on convex sets.

The principal aim of the present paper is to give a generalisation of Santalö's the orem in a different direction. We shall restrict ourselves to intersections by straight lines, but, on the other hand, shall allow much greater freedom in the choice of sets to be intersected. As far as we are aware, our theorems cannot be deduced from Helly's theorem on convex sets.

We shall use the following customary definition:

Two sets $S$ and $S^{*}$ in the plane are said to be separated by a straight line $L$ if $S \subset H \cup L$ and $S^{*} \subset H^{*} \cup L$, where $H$ and $H^{*}, H \neq H^{*}$ are the two open halfplanes determined by $L$. The separation is strict, if $S \subset H$ and $S^{*} \subset H^{*}$.

For the sake of brevity we shall use also the following:

DEFINITION. A family of point sets in the plane is said to have property $d$ if, either (i) there are three sets belonging to the family which cannot be intersected simultaneously by a straight line, or (ii) there exists a straight

Received September 22, 1953. This work was done in a seminar on convex bodies conducted by Prof. A. Dvoretzky at the Hebrew University, Jerusalem.

Pacific. J. Math. 5 (1955), 351-359 
line intersecting all the sets of the family.

Thus, Santalo's theorem is equivalent to the statement that a family of parallel line segments has property $d$.

Our main result is the following:

THE OREM 1. A finite sequence $\left\{S_{i}\right\}(i=1, \cdots, n)$ of connected sets in the plane, such that for every $i=1,2, \cdots, n-1$, there exists a straight line strictly separating the sets $U_{j} \leq i S_{j}$ and $U_{j>i} S_{j}$, has property d.

If the sets $S_{i}$ are compact, this theorem remains valid even for infinite sequences $\left\{S_{i}\right\}(i=1,2, \ldots)$; indeed, we have the somewhat more general theorem:

THEOREM 2. Let $A$ be an ordered set and $\left\{S_{\alpha}\right\}(\alpha \in A)$ a family of compact, connected sets in the plane. If for any finite subset $\left\{\alpha_{1}, \cdots, \alpha_{m} ; \beta_{1}, \cdots, \beta_{m}\right\}$ of $A$, with $\max _{1 \leq i \leq m} \alpha_{i}<\min _{1 \leq j \leq n} \beta_{j}$ (strict inequality) there exists a straight line strictly separating $\cup_{i=1}^{m} S_{a_{i}}$ and $\cup_{j=1}^{n} S_{\beta_{j}}$, then the family $\left\{S_{\alpha}\right\}$ has property d.

If the separating straight lines are parallel, we have stronger results:

THE OREM 3. Every finite family of connected sets in the plane, any two of which can be separated (not necessarily strictly) by a straight line of fixed direction, has property d.

THE OREM 4. Every family of compact, connected sets in the plane, any two of which can be separated (not necessarily strictly) by a straight line of fixed direction, has property d.

In the proofs we shall assume that every three sets may be intersected by a straight line and show the existence of a straight line intersecting all of them.

2. Proof of Theorem 1. 2.1. If $P$ and $Q$ are any two points, we denote by $[P ; Q]$ the closed, by $(P ; Q)$ the open line segment determined by $P$ and $Q$.

We need the following

Lemma. Let $D_{0}$ and $D_{1}$ be two directed straight lines in the plane. For $i=0,1$, let $X_{1}^{(i)}, X_{2}^{(i)}, \ldots, X_{n}^{(i)}$ be a sequence of $n$, not necessarily different, points on $D_{i}$, ordered according to the direction of $D_{i}$ and such that $\left(X_{j}^{(0)}\right.$; $\left.X_{j}^{(1)}\right) \cap\left(X_{k}^{(0)} ; X_{k}^{(1)}\right)=\phi$ for $j \neq k$. ( $\phi$ denotes the void set. $)$ Then there exists 
a family $D(t), 0 \leq t \leq 1$, of straight lines, depending continuausly on $t$, such that

$D(0)=D_{0} ; D(1)=D_{1} ; D(t) \cap\left[X_{j}^{(0)} ; X_{j}^{(1)}\right] \neq \phi \quad(0 \leq t \leq 1 ; j=1,2, \cdots, n)$.

Proof of the Lemma. The Lemma is obvious if either $D_{0}$ and $D_{1}$ are parallel, or

$$
D_{0} \cap\left[X_{1}^{(1)} ; X_{n}^{(1)}\right]=D_{1} \cap\left[X_{1}^{(0)} ; X_{n}^{(0)}\right]=\phi
$$

In the remaining case we may assume $D_{0} \cap\left[X_{1}^{(1)} ; X_{n}^{(1)}\right] \not \phi$. Let $D_{i}^{+}$and $D_{i}^{-}$denote the two open half-lines determined by the intersection point $P=$ $D_{0} \cap D_{1}$ on $D_{i}$, with $D_{i}^{-}$preceding $D_{i}^{+}$according to the direction given on $D_{i}$. The points $X_{j}^{(0)}$ and $X_{j}^{(1)}$ are said to be wrongly paired if either $X_{j}^{(0)} \in D_{0}^{-}$and $X_{j}^{(1)} \in D_{1}^{+}$, or $X_{j}^{(0)} \in D_{0}^{+}$and $X_{j}^{(1)} \in D_{1}^{-}$.

If there are no wrongly paired points the Lemma is obvious, since $D_{0}$ may be rotated about $P$ till it coincides with $D_{1}$.

If there are wrongly paired points, it is easily seen that

(i) they are consecutive, that is, they are $X_{j}^{(0)}, X_{j}^{(1)}$ for $1 \leq k \leq j \leq k+r \leq n$; and

(ii) either $X_{k}^{(0)}=X_{k+1}^{(0)}=\cdots=X_{k+r}^{(0)}$ or $X_{k}^{(1)}=X_{k+1}^{(1)}=\cdots=X_{k+r}^{(1)}$.

Without loss of generality we may assume $X_{k}^{(0)}=\cdots=X_{k+r}^{(0)} \in D_{0}^{-}$. We pass from $D_{0}$ to $D_{1}$ by rotating $D_{0}$ first about $X_{k}^{(0)}$ until it contains the segment $\left[X_{k+r}^{(0)} ; X_{k+r}^{(1)}\right]$ and then about $X_{k+r}^{(1)}$ until it coincides with $D_{1}$. This completes the proof of the Lemma.

2.2. Now we shall prove Theorem 1 under the restrictive assumption that the sets $S_{i}$ are compact and convex.

We shall proceed by induction on the number of sets, $n$. Since the case $n=3$ is trivial, we may assume that the theorem holds for sequences consisting of not more than $n, n \geq 3$, sets, and prove its validity for sequences consisting of $n+1$ sets.

By the induction hypothesis there exist straight lines intersecting all the sets $S_{i}, i=1,2, \cdots, n$. Let $K$ be the set of all the points in the plane through which there passes at least one straight line intersecting all the sets $S_{i}$, $i=1,2, \cdots, n$. Since the $S_{i}$ are compact, $K$ is closed. We shall prove that $K n S_{n+1} \neq \phi$. 
Since the sets $S_{i}$ are convex, it is clear from the assumption that to every straight line intersecting them a direction may be given so that the intersection of the straight line with $S_{j}$ precedes that with $S_{k}$ for $j<k$. From the convexity of the sets $S_{i}$ it follows then by the Lemma that, given two straight lines both intersecting all $S_{i}, i=1,2, \cdots, n$, it is possible to move one of them continuously until it coincides with the other, in such a way that in all intermediate positions, it intersects all the $S_{i}, i=1,2, \cdots, n$.

Let $L_{i}(i=1,2, \cdots, n)$ be straight lines strictly separating the sets $U_{j \leq i} S_{j}$ and $U_{j>i} S_{j}$. For linguistic convenience we shall assume $L_{n}$ vertical and the half-plane $H_{n}$, determined by $L_{n}$ and containing $U_{j} \leq{ }_{n} S_{j}$, situated on the left side of $L_{n}$. We put $I=K n L_{n}$. Now $I \neq L_{n}$ ( since we may assume $L_{1}$ not vertical and then $\left.L_{1} \cap L_{n} \notin I\right)$. Since $I$ is closed, there are in view of the Lemma four possible, mutually exclusive cases:

(a) $l=\phi$;

(b) $I$ is a closed segment (possibly reducing to a single point);

(c) $I$ is a closed half-line;

(d) I consists of two closed, disjoint half-lines.

Case (a). This case may be easily reduced to one of the remaining cases. In fact, we remark:

(i) since $K \neq \phi$, it contains at least one straight line, which is parallel to $L_{n}($ since $l=\phi)$;

(ii) any straight line $L_{n}^{\prime}$, obtained from $L_{n}$ by a sufficiently small rotation about one of its points, separates strictly the sets $S_{n+1}$ and $U_{j \leq n} S_{j}$. But then $K \cap L_{n}^{\prime} \neq \phi$, that is, we have one of the cases (b), (c), (d). (Actually, $K$ consists in case $(a)$ of a single straight line, but we do not use this in our proof.)

Case (b). Let $E$ denote the lower end-point of $l$ and let $T$ denote a straight line, which passes through $E$ and intersects all $S_{i}, i=1,2, \cdots, n$. There exists only one such line $T$ and, moreover, there exist two sets, $S_{p}$ and $S_{q}, p<q$, such that $T$ separates them, $S_{p}$ being contained in the lower closed half-plane determined by $T$.

Indeed, if there were two different straight lines $T_{1}$ and $T_{2}$, through $E$, both intersecting all $S_{i}, i=1,2, \cdots, n$, with $T_{2} \cap H_{n}$ below $T_{1} \cap H_{n}$, the straight line $T_{3}$ passing through any point of $T_{1} \cap S_{1}$ and any point of $T_{2} \cap S_{n}$ would also intersect all $S_{i}, i=1,2, \cdots, n$, but its intersection with $L_{n}$ would be below $E$, in contradiction to the definition of $E$. 
Similarly, at least one $S_{i}$ must be contained in the upper closed half-plane determined by $T$, since otherwise a suitable translate of $T$ would still intersect all the $S_{i}, i=1,2, \cdots, n$, while intersecting $L_{n}$ below $E$ - again a contradiction.

Now let $q$ be the greatest index such that $S_{q}$ has no points below $T$. If all $S_{i}, i<q$, had points above $T$, it would be possible to rotate $T$ about any point of $T \cap S_{q}$ in such a way that, while intersecting all $S_{i}, i=1,2, \ldots, n$, it would intersect $L_{n}$ below $E$-again a contradiction. This proves our statements about $T$.

Similarly we see that through $E^{\prime}$, the upper end-point of $l$, there passes a unique straight line $T^{\prime}$, intersecting all $S_{i}, i=1,2, \cdots, n$, and separating at least two of them, say $S_{p^{\prime}}$ and $S_{q^{\prime}}, p^{\prime}<q^{\prime}$, with $S_{p}$, contained in the upper closed half-plane determined by $T^{\prime}$. Moreover, the slope of $T^{\prime}$ is not smaller than that of $T$, since $T$ is a separating common tangent to $S_{p}$ and $S_{q}$, while $T^{\prime}$ intersects them. (We note that $S_{p}$ and $S_{q}$ may coincide with $S_{p}$, and $S_{q}{ }^{\circ}$ )

$H_{n}^{*}$ being the half-plane to the right of $L_{n}$, its subset $M$ consisting of all the points not above $T^{\prime}$ and not below $T$ is well defined. From the definition of $I$ and the lemma of 2.1 it follows immediately that $K \cap H_{n}^{*} \supset M$. (Actually $K \cap H_{n}^{*}=M$, as may be seen from the sequel, but we do not use this.) The complement of $M$ in $H_{n}^{*}$ consists of two open, disjoint sets, one of them being below, the other above $M$.

Now $S_{n+1} \subset H_{n}^{*}$ and it cannot lie entirely below (resp. above) $M$ since in the first (resp. second) case no straight line would intersect $S_{p}, S_{q}$ (resp. $\left.S_{p}, S_{q},\right)$ and $S_{n+1}$, contradicting the assumption about the existence of a straight line intersecting any three of the sets. But $S_{n+1}$ is connected, therefore $S_{n+1} \cap M \neq \phi$, and since $M \subset K$, this completes the proof in case (b).

Case (c). Assuming the half-line $l$ directed upwards, let $E$ denote its endpoint. As in Case (b), we may prove the existence of a unique straight line $T$ passing through $E$, intersecting all $S_{i}, i=1,2, \cdots, n$, and separating at least two of them, $S_{p}$ and $S_{q}, p<q, S_{p}$ having no points above $T$. Let $M$ be the set of all points to the right of $L_{n}$ and above or on $T$, As in Case (b), $M \subset K$. But $M \cap S_{n+1} \neq \phi$, since otherwise it would be impossible to intersect $S_{p}, S_{q}$ and $S_{n+1}$, in contradiction to the assumptions. Therefore we have $K n S_{n+1} \neq \phi$, completing the proof in Case (c).

Case (d) I consists of two closed, disjoint half-lines on $L_{n}$. Denote by $E_{1}$ and $E_{2}$, with $E_{1}$ below $E_{2}$, the end-points of these half-lines. As in Case (b) we may establish the existence of unique straight lines $T_{j}, j=1,2$, passing 
through $E_{j}$ and intersecting all $S_{i}, i=1,2, \cdots, n$, and separating at least one pair of sets, say $S_{p_{j}}$ and $S_{q_{j}}$, such that $T_{j} \cap S_{q_{j}}$ is nearer to $E_{j}$ than $T_{j} \cap S_{p_{j}}$. It is easy to see that $T_{2}$ has a greater slope than $T_{1}$.

Let $N$ be the subset of $H_{n}^{*}$ consisting of all the points which are either in the lower closed half-plane determined by $T_{1}$, or in the upper one determined by $T_{2}$. As in case (b) we see that $K \cap H_{n}^{*} \supset N$.

Now, $S_{n+1}$ and $U_{i \leq n} S_{n}$ are strictly separated by $L_{n}$. Also, if min $\left(p_{2}, q_{1}\right) \geq$ $\max \left(p_{1}, q_{2}\right)\left(\right.$ resp. $\left.\min \left(p_{1}, q_{2}\right) \geq \max \left(p_{2}, q_{1}\right)\right)$ there exists a straight line - for example, $L_{p_{1}}$ (resp. $\left.L_{p_{2}}\right)$ - strictly separating $S_{p_{1}}$ (resp. $S_{p_{2}}$ ) and $S_{q_{1}} \cup S_{n+1}$ (resp. $\left.S_{q_{2}} \cup S_{n+1}\right)$. Now if $S_{n+1}$ is above $T_{1}$ (resp. below $T_{2}$ ), there cannot exist a straight line intersecting $S_{p_{1}}, S_{q_{1}}$ (resp. $S_{p_{2}}, S_{q_{2}}$ ) and $S_{n+1}$. Hence, by the assumption about the intersectability of any three sets, $S_{n+1}$ must have points in common with $N$, and thus also with $K$. This completes the proof of Theorem 1 for compact and convex $S_{i}$.

2.3. The assumption of compactness of the sets $S_{i}$ may be dropped by a well-known method, used also in [1] in the proof of Helly's theorem.

Suppose it possible to intersect every three of the convex sets $S_{i}$ by a suitable straight line. To every triple of sets $S_{i}$ take one such line and on it one point in each of the three sets. In every set $S_{i}$ a finite number of points is thus obtained. Obviously, the convex hull of these points, $S_{i}^{*}$, is compact and $S_{i}^{*} C$ $S_{i}, i=1,2, \cdots, n$. The sequence $\left\{S_{i}^{*}\right\}$ clearly satisfies all the assumptions of 2.2 , so that we can conclude that there exists a straight line intersecting all $S_{i}^{*}$, and, a fortiori, all the sets $S_{i}$.

2.4. Finally, we drop the assumption of convexity by the simple remark that, if two sets are (strictly) separated by a straight line, so are their convex hulls, and if a straight line intersects the convex hull of a connected set, it intersects the set itself as well. We can, therefore, from a given sequence of connected sets pass to the sequence of their convex hulls, and after applying to these the result of 2.3 , return to the sets themselves.

This completes the proof of Theorem 1.

3. Proof of Theorem 2. Let $\alpha_{1}, \beta_{1} \in A$ with $\alpha_{1}<\beta_{1}$. There exists a straight line strictly separating $S_{a_{1}}$ and $S_{\beta_{1}}$. Following a method used in [1] we introduce in the plane an orthogonal coordinate system $X Y$ in such a way that the $Y$ axis strictly separates $S_{\alpha_{1}}$ and $S_{\beta_{1}}$. To every straight line $y=$ $\xi x+\eta$ in the $X Y$ plane corresponds the point $(\xi, \eta)$ in another, $\Xi H$, plane. 
For any finite subset $\left\{\alpha^{\prime}\right\}$ of $A$, it follows from Theorem 1 that there exist straight lines intersecting all $S_{\alpha}$. If $\left\{\alpha_{1}, \beta_{1}\right\} \subset\left\{\alpha^{\prime}\right\}$ then the set of points $(\xi, \eta)$ corresponding to all such straight lines is a compact set in the $\Xi H$ plane. Since the intersection of any finite number of such sets is not void, it follows from a well-known theorem of $F$. Riesz that the intersection of all of them is not void. But a straight line corresponding to a point common to all these sets intersects all the sets $S_{a}, \alpha \in A$. Q.e.d.

4. Proof of Theorem 3. Without loss of generality we may assume that the straight lines separating the sets are vertical.

As in the proof of Theorem 1 we assume the sets $S_{i}$ convex and compact. It is easy to get rid of these restrictions exactly as in 2.3 and 2.4. Our proof is again inductive, we assume the theorem for some $n \geq 3$ and prove it for $n+1$.

Every one of the $n+1$ sets is contained in a minimal strip, bounded by two vertical straight lines (the two lines may coincide or one of them may be at infinity). Strips containing different sets have an intersection which is either void or consists of a single vertical line belonging to the boundary of the strips.

It is possible, therefore, to enumerate the sets proceeding, say, from left to right and, in case of indetermination - that is, when a vertical line contains more than one set, - in an arbitrary fashion. Let $V_{i}$ be a vertical line separating the sets $S_{i}$ and $S_{i+1}, i=1,2, \cdots, n$. It follows from the method of enumeration that $V_{i}$ separates $U_{j} \leq i S_{j}$ and $U_{j}>_{i} S_{j}$.

If for some $s, 1 \leq s \leq n-2, V_{s} \equiv V_{s+2}$, the sets $S_{s+1}$ and $S_{s+2}$ are situated entirely on the straight line $V_{s} \equiv V_{s+1} \equiv V_{s+2}$. Then either

(i) $S_{s+1} \cap S_{s+2}=\phi$ and $V_{s}$ is the only straight line intersecting $S_{s+1}$ and $S_{s+2}$, hence, since every three sets can be intersected by a straight line, all the sets $S_{i}$ have to intersect $V_{s}$ and the theorem is proved; - or

(ii) $S^{\prime}=S_{s+1} \cap S_{s+2} \neq \phi$ and then it is easily seen (either directly or by the Lemma of 2.1) that the $n$ sets $S_{1}, \cdots, S_{s}, S^{\prime}, S_{s+3}, \cdots, S_{n+1}$ satisfy all the conditions of the theorem so that, by the induction hypothesis, there exists a straight line intersecting all of them, hence, a fortiori, intersecting all $S_{i}, i=1,2, \cdots, n+1$.

Thus we may assume $V_{i} \neq V_{i+2}, i=1,2, \cdots, n-2$, therefore a straight line intersecting all the sets $S_{i}, i=1,2, \ldots, n$, cannot be vertical, hence it must intersect $V_{n}$. Let $K$ denote the set of all points through which there pass straight lines intersecting all $S_{i}, i=1,2, \cdots, n$. From the lemma of 2.1 it follows that $l=K \cap V_{n}$ is a closed segment (possibly reducing to a point). Using the 
same method as in the proof of Case (b) of Theorem 1 we may see that there exist straight lines $T$ and $T^{\prime}$ and sets $S_{p}, S_{p^{\prime}}, S_{q}, S_{q^{\prime}}$, having all the properties of the sets so denoted in 2.2. As in Case (b) of Theorem 1 it follows that $S_{n+1}$ must contain points not below $T$ and not above $T^{\prime}$ and hence that $S_{n+1} \cap K \neq$ $\phi$, completing the proof of Theorem 3 .

5. Proof of Theorem 4. We again assume the separating straight lines vertical. By Theorem 3 to every finite subfamily of the family $\left\{S_{\alpha}\right\}_{(\alpha \in A)}$ there corresponds a straight line intersecting all sets of the subfamily. If to every finite subfamily there corresponds a vertical intersecting line, it is easily seen that all the sets $S_{a}$ (except possibly one or two), lie entirely on one vertical line (which intersects also the possibly remaining one or two) and the theorem is established in this case.

If there is at least one finite subfamily of $\left\{S_{a}\right\}$ for which there is no vertical straight line intersecting all the sets of the subfamily, then there exist two sets $S_{a_{1}}$ and $S_{\beta_{1}}$ in the subfamily, which can be separated by a vertical straight line. We then complete the proof exactly in the way in which we proved Theorem 2.

6. Remarks. Simple examples show that some of the assumptions made in Theorems $1-4$ are essential.

6.1. The connectedness of the sets $S_{i}$ required in the theorems cannot be dropped entirely. Indeed, denoting by $[P ; Q]$ the closed segment with endpoints $P$ and $Q$, let

$$
\begin{array}{ll}
S_{1}=[(0,0) ;(0,3)], & S_{2}=[(1,0) ;(1,2)], \\
S_{3}=(2,3) \cup[(2,0) ;(2,1)], & S_{4}=[(3,2) ;(3,3)] .
\end{array}
$$

Then, except that $S_{3}$ is not connected, the sequence $S_{1}, S_{2}, S_{3}, S_{4}$ satisfies the assumptions of all four theorems and yet it does not have property $d$.

It is possible however to substitute for the assumption of connectedness the somewhat weaker one that if both open half-planes determined by a straight line $L$ contain points of $S$ then $L \cap S \neq \phi$. As a matter of fact, in 2.4 and the corresponding part of the proof of Theorem 3 only the above property of connected sets was used.

6.2. The strict separation required in Theorems 1 and 2 cannot be dropped. Indeed, let 


$$
\begin{aligned}
& S_{1}=[(-3,-1) ;(0,-1)], S_{2}=[(-1,0) ;(-1,1)], \\
& S_{3}=[(1,0) ;(1,1)], \quad S_{4}=[(0,-1) ;(3,-1)] .
\end{aligned}
$$

Except for the impossibility to separate strictly $S_{1}$ and $S_{4}$, the sequence $S_{1}$, $S_{2}, S_{3}, S_{4}$ satisfies all the assumptions of Theorems 1 and 2, although it does not have property $d$.

6.3. The compactness assumption in Theorems 2 and 4 cannot be dropped either. Indeed, if $S_{0}$ is the open interval with endpoints $(0,0)$ and $(0,1)$, and $S_{i}=[(i, 0) ;(i, 1 / i)], i=1,2,3, \cdots$, the sequence $\left\{S_{i}\right\}(i=0,1,2, \ldots)$ satisfies all the assumptions of Theorems 2 and 4 except for the noncompactness of $S_{0}$, and yet it does not have property $d$.

Added in proof. Since the submission of this paper a slightly weaker version of Theorems 3 and 4 appeared in a paper by V.L. Klee, Jr., Common secants for plane convex sets, Proc. Amer. Math. Soc., 5 (1954), 639-641.

\section{REFERENCES}

1. H. Rademacher and I. J. Schoenberg, A proof of Helly's theorem on convex bodies and Tchebycheff's approximation problem, Canad. Journal of Math. II., (1950), 245-256.

2. L.A. Santaló, Supplement to the note: A theorem on sets of parallelepipedes with parallel edges, Publ. Inst. Mat. Univ. Nac. Litoral, 3 (1942), 202-210. (Spanish)

\section{HEBREW UNIVERSITY}

JER US A LEM 



\section{PACIFIC JOURNAL OF MATHEMATICS}

\section{EDITORS}

\author{
H.L. ROYDEN \\ Stanford University \\ Stanford, California \\ E. HEWITT \\ University of Washington \\ Seattle 5, Washington
}

\author{
R. P. DILWORTH \\ California Institute of Technology \\ Pasadena 4, California \\ * Alfred Horn \\ University of California \\ Los Angeles 24, California
}

\section{ASSOCIATE EDITORS}

\section{H, BUSEMANN \\ HERBERT FEDERER}

MARSHALL HALL

\section{P.R. HALMOS \\ HEINZ HOPF}

ALFRED HORN

\author{
R.D. JAMES \\ BØRGE JESSEN \\ PAUL LÉVY
}

GEORGE PÓLYA

J.J. STOKER

KOSAKU YOSIDA

\section{SPONSORS}

UNIVERSITY OF BRITISH COLUMBIA

UNIVERSITY OF SOUTHERN CALIFORNIA

CALIFORNIA INSTITUTE OF TECHNOLOGY

UNIVERSITY OF CALIFORNIA, BER KELEY

STANFORD RESEARCH INSTITUTE

STANFORD UNIVERSITY

UNIVERSITY OF CALIFORNIA, DAVIS

UNIVERSITY OF. UTAH

UNIVERSITY OF CALIFORNIA, LOS ANGELES

WASHINGTON STATE COLLEGE

UNIVERSITY OF CALIFORNIA, SANTA BARBARA

UNIVERSITY OF WASHINGTON

MONTANA STATE UNIVERSITY

UNIVERSITY OF NEVADA

OREGON STATE COLLEGE

AMERICAN MATHEMATICAL SOCIETY

HUGHES AIRCRAFT COMPANY

UNIVERSITY OF OREGON

SHELL DEVELOPMENT COMPANY

\section{UNIVERSTTY OF SOUTHERN CALIFORNIA}

Mathematical papers intended for publication in the Pacific Journal of Mathematics should be typewritten (double spaced), and the author should keep a complete copy. Manuscripts may be sent to any of the editors. Manuscripts intended for the outgoing editors should be sent to their successors. All other communications to the editors should be addressed to the managing editor, Alfred Horn, at the University of California Los Angeles 24, California.

50 reprints of each article are furnished free of charge; additional copies may be obtained at cost in multiples of 50 .

The Pacific Journal of Mathematics is published quarterly, in March, June, September, and December. The price per volume (4 numbers) is $\$ 12.00$; single issues, $\$ 3.50$; back numbers (Volumes $1,2,3$ ) are available at $\$ 2.50$ per copy. Special price to individual faculty members of supporting institutions and to individual members of the American Mathematical Society: $\$ 4.00$ per volume; single issues, $\$ 1.25$.

Subscriptions, orders for back numbers, and changes of address should be sent to the publishers, University of California Press, Berkeley 4, California.

Printed at Ann Arbor, Michigan. Entered as second class matter at the Post Office, Berkeley, California.

* During the absence of E.G. Straus.

UNIVERSITY OF CALIFORNIA PRESS - BERKELEY AND LOS ANGELES 


\section{Pacific Journal of Mathematics}

\section{Vol. 5, No. $3 \quad$ November, 1955}

Nesmith Cornett Ankeny and S. Chowla, On the divisibility of the class number of quadratic fields ............................. 321

Cecil Edmund Burgess, Collections and sequences of continua in the plane ........................................ 325

Jane Smiley Cronin Scanlon, The Dirichlet problem for nonlinear elliptic equations....................................... 335

Arieh Dvoretzky, A converse of Helly's theorem on convex sets ......... 345

Branko Grünbaum, On a theorem of L. A. Santaló................ 351

Moshe Shimrat, Simple proof of a theorem of P. Kirchberger .......... 361

Michael Oser Rabin, A note on Helly's theorem . ................... 363

Robert E. Edwards, On factor functions . . ................... 367

Robert E. Edwards, On certain algebras of measures ............... 379

Harley M. Flanders, Methods in affine connection theory.............. 391

Alfred Huber, The reflection principle for polyharmonic functions ........ 433

Geoffrey Stuart Stephen Ludford, Generalised Riemann invariants ....... 441

Ralph Gordon Selfridge, Generalized Walsh transforms............. 451 